\title{
(C) OPEN ACCESS \\ A qualitative analysis of the elements used by palliative care clinicians when formulating a survival estimate
}

\author{
Rose Clarkson, Debbie Selby, Jeff Myers
}

Palliative Care Consult Team, Sunnybrook Health Sciences Centre, Toronto, Ontario, Canada

\section{Correspondence to}

Dr Jeff Myers, Palliative Care Consult Team, Sunnybrook Health Sciences Centre, Rm H336, 2075 Bayview Avenue, Toronto, ON, Canada, M4N 3M5;

jeff.myers@sunnybrook.ca

Accepted 22 October 2012 Published Online First 30 November 2012

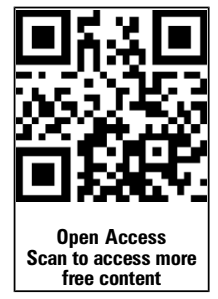

To cite: Clarkson $R$, Selby $D$, Myers J. BMJ Supportive \& Palliative Care 2013;3: 330-334.

\section{ABSTRACT}

Objective For patients with advanced and/or incurable disease, clinicians are often called upon to formulate and communicate an estimate of likely survival duration. The objective of this study was to gain a deeper appreciation of this process by identifying and exploring the specific elements that may inform and/or impact a clinician's estimate of survival (CES).

Methods Semistructured interviews were conducted among a group of palliative care clinicians in the setting of a tertiary academic health sciences centre. Qualitative data were subsequently analysed using a grounded theory approach.

Results Five major themes were identified as being central to the process of CES formulation: use of objective patient-specific elements, strength of the patient-clinician relationship, purpose and context of an individual CES, perceived role of hope and the overall likelihood of CES inaccuracy.

Conclusions For any given patient, several elements have the potential to inform and/or impact the process of CES formulation. Study participants were aware of objective clinical factors known to correlate with actual survival duration and likely integrate this information when formulating a CES. Formulation occurs within a larger context comprised of a number of elements that may influence individual estimates. These elements exist against a background of awareness of the overall likelihood of CES inaccuracy. Clinicians are encouraged to develop a personalised and standardised approach to CES formulation whereby an awareness of the menu of potentially impacting elements is consciously integrated into an individual process.

\section{INTRODUCTION}

A clinician's estimate of likely survival (CES) duration has been described as a subjective process in which a 'personal clinical judgement' is formulated. ${ }^{1}$ Numerous reports have demonstrated that CES is most often inaccurate with a substantial tendency to overestimate. ${ }^{2}$

Despite this, CES has consistently been shown to be an independent predictor of actual survival duration ${ }^{3-6}$ with the suggestion made that CES be used as the reference standard for evaluating other methods of survival estimation. ${ }^{3}$ The European Association for Palliative Care recently published evidence-based clinical recommendations regarding overall methods and processes used in survival estimation. ${ }^{7}$ One of six key outcomes was the recommendation that CES be accepted as a valid process. Clinicians were cautioned, however, to be aware of elements impacting accuracy.

With the overall aim of gaining a deeper understanding of CES formulation, the primary objective for the current study was to identify and explore the specific elements that may inform and/or impact the process of CES formulation among palliative care clinicians.

\section{METHODS}

\section{Research design and participants}

Individual semistructured interviews were completed for this qualitative study. Potential participants were members of a consultative palliative care team within a tertiary academic health sciences centre. Purposive sampling was used to select the physicians and advanced practice nurses who responded to a general emailed invitation and subsequently were offered formal study participation. Of note, clinicians routinely formulate a CES for every patient referred for palliative care 
consultation in outpatient and inpatient settings. This is done at the time of initial assessment irrespective of the reason for referral and is represented by the choice of one time-based prognostic category felt to best represent an individual patient's likely survival duration. ${ }^{2}$ Clinicians were aware the CES data was being collected purely for administrative and academic purposes and in general, individual CES formulations were neither expected to be communicated to patients nor impact clinical decision-making. In addition, at the time of the present study, feedback had not yet been given to the clinicians regarding individual rates of CES accuracy.

Separate interviews were completed for individual participants and informed consent and demographic information was obtained prior to the start of each. An interview guide was developed and field-tested to ensure clarity of the questions. The open-ended questions allowed clinicians to identify and explore factors perceived as possibly influencing the process of CES formulation. The interviewer used directive probes to elicit additional information and clarify responses. To capture possible attitudinal, personality or valuesbased factors, clinicians were asked a series of questions that included previous experiences with survival estimation, perception of general estimate accuracy and possible circumstances of a conscious choice to either overestimate or underestimate. Field notes were kept to capture key points and observations made during the interviews.

\section{Ethical considerations}

Ethics approval was obtained from the Research Ethics Board of the Sunnybrook Heath Sciences Centre.

\section{Data analysis}

Interviews were audio-recorded and transcribed verbatim. Descriptive data (ie, transcripts and the accompanying field notes) were analysed using a grounded theory approach.

Specifically, data was coded in a line-by-line process through which keywords, phrases and texts were identified. Concepts were subsequently organised by literal and interpretive meanings and grouped thematically into categories. Key categories and concepts were compared iteratively looking for similarities, differences and relationships, then organised into broader themes. Major themes were then organised into a theoretical framework by making first degree connections between the categories. To maximise validity, two investigators analysed the data separately and agreement for the major themes was achieved through an iterative process and comparative analyses. Minor disagreements were resolved through discussion and exploration of the choice of terminology.

\section{RESULTS}

In total, nine interviews were conducted including those of seven physicians and two advanced practice nurses. The entire clinical practice for all participants was focused on palliative care with a range from 4-14 years for the physicians and 8-9 years for the nurses.

Clinicians provide palliative care in inpatient and ambulatory clinic settings for patients with a range of diagnoses and actual survival duration.

Five major themes were identified as being central to the process of CES formulation:

1. Use of objective patient-specific elements

All participants identified certain objective measures that they in some way use when estimating survival duration. Performance status, as an example, was cited by all participants as being critical to CES formulation:

As a palliative care physician, we're looking at performance status.

Additionally, patient demographic information was identified as being of particular importance:

The frail elderly person...I am more likely to give a lower prognosis than a young healthy person who I think...may survive longer.

Certain biochemical markers (eg, albumin, creatinine and $\mathrm{C}$ reactive protein) as well as specific symptoms and signs (eg, 'weight loss', 'dsypnoea' and 'delirium') were felt likely to contribute to a CES formulation, particularly among actively dying patients. For patients living with cancer, the specific type, stage and grade were noted to be important factors:

...if somebody's full of liver mets and brain mets, that's a pretty hard finding

Most clinicians spontaneously introduced survival estimation tools or models into the discussion, conveying the general opinion that they are of limited practical value when applied to individual patients:

There are references that can guide you, that will give you means and medians and standard deviations. So, that's always...in the back of your mind, the stuff that we theoretically know. But that's a statistic.

2. The patient-clinician relationship

The strength of the relationship between the clinician and patient was viewed by all participants as an important element of CES formulation and had the potential to impact the process, either consciously or subconsciously. Several participants acknowledged the possibility of overestimating survival among patients with whom they had a particularly strong relationship:

...if I develop a rapport or connection with a patient that's particularly strong... would that impact how I see their prognosis, and might I extend that because of 
my wish? That this person should live longer? And in that case I would say yes, I think it probably does.

I'm human, right? So I'm going to connect with certain patients more than others. For those that I have a connection with, feel that personal drive, to want them to live. So my discussions probably are going to be biased in a way that is going to make them lean more towards being more optimistic about their prognosis. I admit that. I try and be as objective as I can be, but I can't deny that I'm going to be human and do that.

I'm aware of it, and I try and be conscious of it, but I know in my mind, with patients I have better relationships with, I struggle with giving them a poor prognosis.

The element of trust within the clinician-patient relationship, in particular its maintenance, was viewed as being important. The potential consequence of an inaccurate CES being a loss of this trust was identified as potentially impacting the CES formulation process:

I don't worry that I'm going to give the patient a poor prognosis and they're going to have a different perception of our doctor/physician-patient relationship. The concern is, if I'm inaccurate, does that affect their trust in me as their physician?

3. Purpose and context of an individual CES

The underlying purpose for any given CES and how it might be used was identified as a possible element contributing to the process of CES formulation. In particular, participants outlined the potential impact on the intended audience that is, patient, family members, the clinician themselves (ie, the individual formulating the CES), other health professionals or allocators of healthcare resources. A specific example cited by several participants was the circumstance in which the CES determines qualification for access to certain palliative care services:

Maybe I might want to underestimate. And also then they get some more services in terms of palliative care services in the community.

In exploring the idea that communicating any given CES to a patient may then become a 'self-fulfilling prophecy', nurses and physicians differed in their response. Both nurses and physicians indicated their belief in this being an important phenomenon of which clinicians should be mindful:

I think for some people, if you say it's going to be, they believe it. Especially if they trust you... I've seen people really just become deflated when they hear that.

The physician participants uniformly disagreed with the notion that communicating a CES might impact actual survival duration. All physicians however did acknowledge 'self-fulfilling prophecies' as being a common source of distress for family members, thus requiring insight into patient/family perspectives regarding this topic:

Families are more concerned if you give them (patients) this prognosis, they'll die. Or they'll lose their will to live. I see that in, a lot, in families. Less so in patients. I get the sense that patients are in general more realistic and want to know more information than their families think.

4. Perceived role of hope

A desire to maintain a perceived sense of hope was frequently identified as possibly influential on a CES formulation. The importance of maintaining hope was balanced however with a perceived professional duty to avoid what was described as 'giving a false sense of hope':

I'm not there to take away a patient's hope to live, and a drive to live

I'm the type of person that likes to try and give patients hope and give them kernels of hope, but realistic hope. So I don't like giving people false hope. So I don't want to say that I'd like my estimates to be overly optimistic, I think that's giving people false hope.

\section{Likelihood of CES inaccuracy}

All participants rated the accuracy of their own survival estimates and those of healthcare providers in general as being poor. Frequently addressed was the notion of impossibility for any CES to be precisely accurate:

It's about the same as flipping a coin

I'm pretty average compared with other physicians. I wouldn't say that I'm better or worse than other physicians. I think we're all sort of in the same boat

Do I ever think anything will be $100 \%$ accurate? Absolutely not.

One participant indicated she endeavoured to balance the likelihood of inaccuracy by consciously underestimating:

If the prognosis is just relevant to me, I would rather underestimate it...I am going to try as best I can to prepare the patient and family for what is to come, and that I have the time to do that. And that the rug isn't pulled out from under all of us by me overestimating.

Participants alluded to overall limitations in human knowledge and the epistemic constraints impacting the timing of death. This was usually framed in terms of a fundamental mystery regarding death, one that scientific inquiry could not possibly resolve:

So I wonder, either we just haven't found the right tool or tools, or perhaps there's something around the end-of-life that will always be...unscientific. 
So I think there's that thing, that kind of core to the person that we can't ultimately affect... That's the mystery of human life and human death. And so no matter how skilled we become, I don't think that we're going to ultimately be able to call everyone.

At various points throughout each interview, participants' perspective on the importance of skilful CES communication was outlined.

\section{DISCUSSION}

A recent 'State of the Science' meeting involving expert clinicians and researchers in prognostication was held with the aim of identifying and defining key research priorities for the field. ${ }^{8}$ Of the 40 specific topics considered, 'what clinicians use and what they are considering when estimating survival' was identified as the second most important priority. In seeking to understand the clinicians' perceived elements contributing to the process of CES formulation, the results of the present study outline an initial understanding of one aspect of this key priority.

The main findings in this study provide a preliminary overview of several factors that may impact CES formulation for any given patient. To summarise, palliative care clinicians are aware of objective clinical factors known to correlate with actual survival duration and likely integrate this information when formulating a CES. In addition, the CES for an individual patient is formulated within a larger context comprised of a number of elements that may influence individual estimates. These include the relationship the clinician has with the patient and the patient's family, the intent and audience for the CES and the overall likelihood of inaccuracy, if a certain level of precision is the expectation. Finally, the personal belief system and set of values of a clinician, in particular any perceived need to preserve a sense of hope among patients and family members, may impact the process of CES formulation.

For clinicians in this study, the importance of communication was identified and underscored as a critical component of CES. Whether or not some element of the CES communication process might influence the formulation process remains unclear.

Historically, survival estimation has been categorised into two general methods. In the first method, CES, has been summarised as a 'personal clinical judgement' that is subjective in nature with complex and poorly defined variables. ${ }^{1}$ Pontin and Jordan recently reported the findings of their study addressing views on the overall prognostication experience among a cohort of palliative care clinicians. ${ }^{9}$ The study provides an important overview of the clinicians' experience and particularly underscores the potential impact of CES inaccuracy on clinicians themselves and the fear and uncertainty often experienced. The authors indicate clinicians appear to use certain prognostic factors in prognostication' and go on to identify that 'further work is required to identify how and why these factors are used'. One of the strengths of the current study is the participants' ability to reflect on the CES-related process itself that is, routine formulation for each patient upon initial assessment, without either a standard expectation of CES communication or linkage to clinical decision-making. The resulting team culture with respect to CES overall is such that clinicians are able to formulate without substantial concern for any direct impact on patients and families.

The second method of survival estimation is comprised of objective and actuarial measures typically integrated into some form of a prognostic tool or formula, whereby an estimate is generated from the amalgamation of clinical data elements and related mortality data.

Previously identified clinical indices known to correlate with actual survival duration include patient performance status, presence of anorexia and/or weight loss, presence of breathlessness and/or delirium and certain biological parameters (eg, serum albumin, white blood cell count and lymphocyte ratio). ${ }^{7}{ }^{10-13}$ The finding in the present study that clinicians may consciously or subconsciously consider objective patient-specific measures when formulating a CES, outlines an objective component of the largely subjective process. The idea that during CES formulation clinicians may integrate objective measures known to correlate with actual survival duration highlights what is likely to be an overlap between the two general methods of survival estimation, previously outlined as separate subjective and objective processes.

The length of the clinician-patient relationship has previously been reported to directly correlate with CES accuracy that is, the longer the duration, the lower the accuracy. ${ }^{14}$ Although 'length' is somewhat different than 'strength' in the context of interpersonal relationships, by acknowledging the likelihood of CES overestimation for patients with whom they have a stronger relationship in comparison to others, participants in the present study qualitatively validate Christakis' finding. Consciously acknowledging the 'strength' of the clinician-patient relationship and proactively factoring this element into a CES formulation may at least in part correct for its impact on the resulting CES itself. Similarly, a conscious acknowledgement could be given to each of the potentially influential elements identified by study participants, which include intended audience, implications for resource allocation and clinicians' personal tendencies, values and belief systems.

A number of limitations exist in the present study. First, study participants were from one institution, thus limiting generalisability. Second, since the given study participants were all full time palliative care clinicians, we did not capture the perspective of other 
disciplines with respect to possible elements impacting CES formulation. Another important limitation of the study relates to participants only being able to identify potentially influential elements of which they were consciously aware. As with any individual decisionmaking process, a range of elements have the potential to have a subconscious impact. The elements generated from this study may not be comprehensive or exhaustive and the interaction between elements not accounted for. Future research should include work that is similar, completed at different centres and among different fields of healthcare. Additionally, in having gained a better understanding of the elements comprising a CES, efforts could be focused on designing, implementing and evaluating a standard approach to CES formulation and examining the impact on patients and family members for whom the formulation is communicated.

\section{CONCLUSION}

Multiple 'calls to action' have been made for the development and/or refinement of survival estimate tools that provide more accurate information. Results of the current study suggest parallel efforts should be made towards encouraging clinicians to develop a personalised and standardised approach to CES formulation whereby an awareness of the menu of potentially impacting elements are consciously integrated into an individual process. In addition, it may be wise to acknowledge the ultimate impossibility of absolute accuracy in survival estimation and ensure that efforts are also focused on developing and disseminating a standardised approach to CES communication. This includes identifying effective professional development strategies such that clinicians would achieve and maintain competency in communicating survival estimation in a manner that is individualised and meaningful.

Contributors Conception and design: JM and RC. Data acquisition and analysis: JM and RC. Data interpretation: JM, RC and DS. Development of manuscript draft: RC. Manuscript revision: JM, RC and DS. Final approval of submission: JM, RC and DS. Guarantor responsible for overall content: JM.

\section{Funding None.}

\section{Competing interests None.}

Ethics approval Sunnybrook Health Sciences Centre IRB.

Provenance and peer review Not commissioned; externally peer reviewed.

Open Access This is an Open Access article distributed in accordance with the Creative Commons Attribution Non
Commercial (CC BY-NC 3.0) license, which permits others to distribute, remix, adapt, build upon this work

non-commercially, and license their derivative works on different terms, provided the original work is properly cited and the use is non-commercial. See: http://creativecommons. org/licenses/by-nc/3.0/

\section{REFERENCES}

1 Glare P. Predicting and communicating prognosis in palliative care. BMJ 2011;343:d5171.

2 Selby D, Chakraborty A, Lilien T, et al. Clinician accuracy when estimating survival duration: the role of the patient's performance status and time-based prognostic categories. J Pain Symptom Manage 2011;42:578-8.

3 Glare P, Virik K, Jones M, et al. A systematic review of physicians' survival predictions in terminally ill cancer patients. BMJ 2003;327:

195-8.

4 Glare P, Sinclair C, Downing M, et al. Predicting survival in patients with advanced disease. Eur J Cancer 2008;44:1146-56.

5 Mackillop WJ, Quirt CF. Measuring the accuracy of prognostic judgments in oncology. J Clin Epidemiol 1997;50:21-9.

6 Vigano A, Dorgan M, Bruera E, et al. The relative accuracy of the clinical estimation of the duration of life for patients with end of life cancer. Cancer 1999;86:170-6.

7 Maltoni M, Caraceni A, Brunelli C, et al. Prognostic factors in advanced cancer patients: evidence-based clinical recommendations - a study by the Steering Committee of the European Association for Palliative Care. J Clin Oncol 2005;23:6240-8.

8 Stevinson C, Preston N, Todd C. Cancer Experiences Collaborative (CECo). Defining priorities in prognostication research: results of a consensus workshop. Palliat Med 2010;24:462-8.

9 Pontin D, Jordan N. Issues in prognostication for hospital specialist palliative care doctors and nurses: a qualitative inquiry. Palliat Med Published Online First: 21 December 2011. doi:10.1177/0269216311432898

10 Gripp S, Moeller S, Bolke E, et al. Survival prediction in terminally ill cancer patients by clinical estimates, laboratory tests, and self-rated anxiety and depression. J Clin Oncol 2007;25:3313-20.

11 Lau F, Downing M, Lesperance M, et al. Use of palliative performance scale in end-of-life prognostication. J Palliat Med 2006;9:1066-75.

12 Maltoni M, Pirovano M, Nanni O, et al. Prognostic factors in terminal cancer patients. Eur J Palliat Care 1994;1:122-5.

13 Kikuchi N, Ohmori K, Kuriyama S, et al. Survival prediction of patients with advanced cancer: the predictive accuracy of the model based on biological markers. J Pain Symptom Manage 2007;34:600-6.

14 Christakis NA, Lamont EB. Extent and determinants of error in doctors' prognoses in terminally ill patients: prospective cohort study. BMJ 2000;320:469-72. 